\title{
LV-SY-1-2
}

\section{Liver transplantation for acute on chronic liver disease; when and how?}

\author{
Jun Yong PARK*
}

Department of Internal Medicine, Yonsei University College of Medicine, Seoul, Korea

Lecture: Acute-on-chronic liver failure (ACLF) is a syndrome characterized by acute decompensation of chronic liver disease associated with organ failures and high short-term mortality. Alcohol and chronic viral hepatitis are the most common underlying liver diseases. Up to $40 \%-50 \%$ of the cases of ACLF have no identifiable trigger; in the remaining patients, sepsis, active alcoholism and relapse of chronic viral hepatitis are the most common reported precipitating factors. An excessive systemic inflammatory response seems to play a crucial role in the development of ACLF. Using a liver-adapted sequential organ assessment failure score, it is possible to triage and prognosticate the outcome of patients with ACLF. When evaluating prognosis in patients with ACLF, it should be noted that this is a dynamic syndrome that may improve or worsen during hospitalization. Therefore, the ideal scoring system should be able to reflect the dynamic nature of the disease and the responsiveness to medical treatment. Considering the scoring systems and recent data indicating that sequential assessment of prognosis seems to have higher accuracy than prognosis evaluated at the diagnosis of ACLF, stepwise algorithms have been proposed to assess prognosis and help decision making in ACLF patients. 